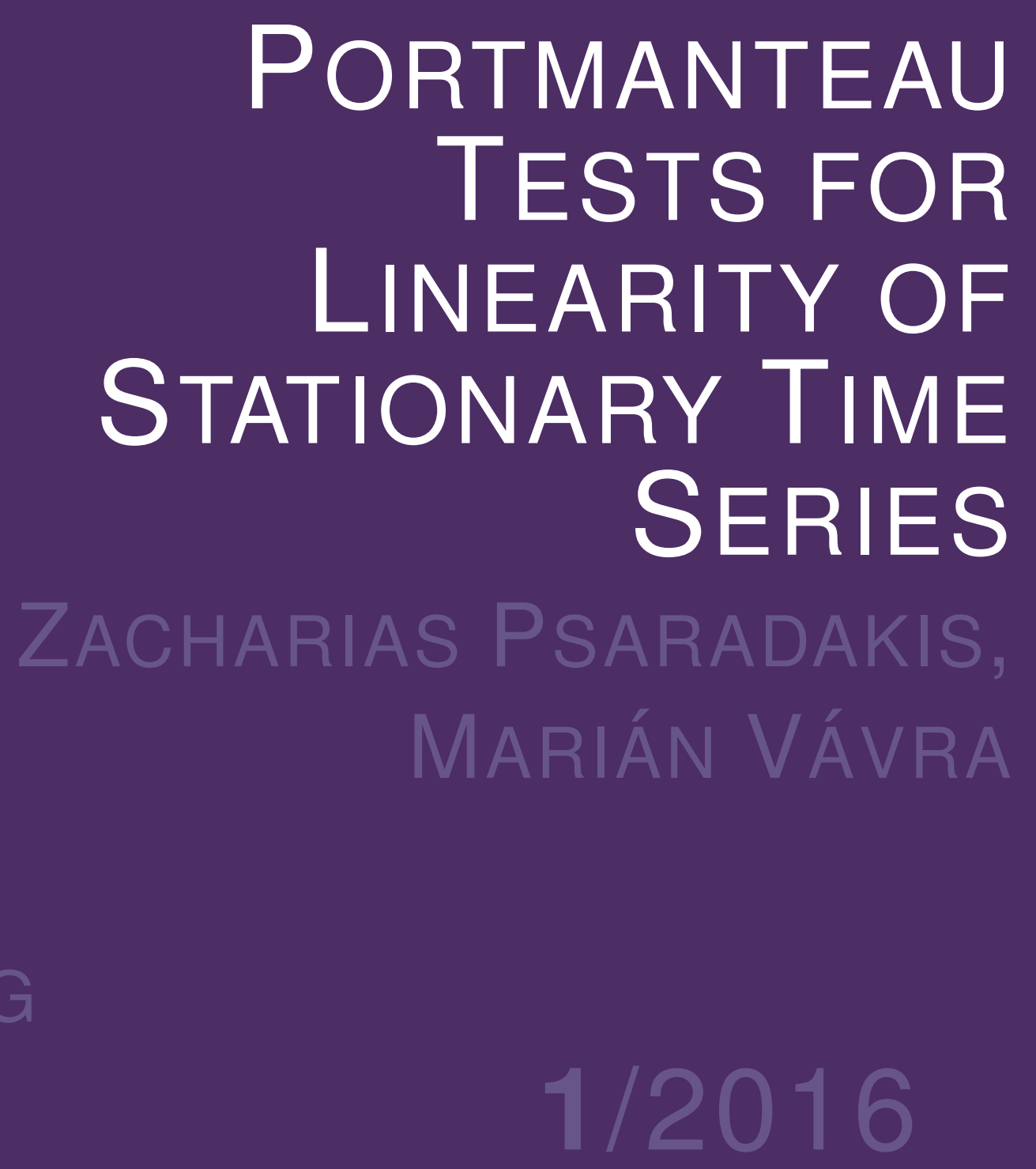


(C) National Bank of Slovakia

www.nbs.sk

Imricha Karvaša 1

81325 Bratislva

research@nbs.sk

April 2016

ISSN $1337-5830$

The views and results presented in this paper are those of the authors and do not necessarily represent the official opinions of the National Bank of Slovakia.

All rights reserved. 


\title{
Portmanteau Tests for Linearity of Stationary Time Series ${ }^{1}$
}

\author{
Working paper NBS
}

Zacharias Psaradakis, Marián Vávra²

\begin{abstract}
This paper considers the problem of testing for linearity of stationary time series. Portmanteau tests are discussed which are based on generalized correlations of residuals from a linear model (that is, autocorrelations and cross-correlations of different powers of the residuals). The finite-sample properties of the tests are assessed by means of Monte Carlo experiments. The tests are applied to 100 time series of stock returns.
\end{abstract}

JEL classification: C12; C22; C52.

Key words: Autocorrelation; Cross-correlation; Nonlinearity; Portmanteau test; Stock returns.

Downloadable at http://www.nbs.sk/en/publications-issued-by-the-nbs/research-publications

\footnotetext{
${ }^{1}$ We would like to thank Ron Smith and participants in the Research Seminar at the NBS for useful comments and interesting suggestions. All remaining errors are only ours.

${ }^{2}$ Zacharias Psaradakis, University of London; Marián Vávra, Research Department of the NBS.
} 


\section{INTRODUCTION}

The problem of testing for neglected nonlinearity in time series models has attracted a great deal of interest in recent years. A multitude of statistical procedures designed to test the null hypothesis of linearity against nonlinear alternatives are available in the literature, including general portmanteau tests without a specific alternative as well as tests with fully specified parametric alternatives; Tong (1990) and Teräsvirta, Tjøstheim, and Granger (2010) provide useful overviews. Linearity tests have become an essential first step in model-building exercises since, due to the difficulties associated with the statistical analysis of nonlinear models, it is often desirable to establish the adequacy or otherwise of a linear data representation before exploring more complicated nonlinear structures.

The present paper contributes to this literature by considering portmanteau tests for linearity of stationary time series based on 'generalized correlations' of residuals from a finite-parameter linear model, that is to say autocorrelations and cross-correlations of different powers of the residuals. Such tests are similar in spirit to the popular test proposed by McLeod and Li (1983), which is based on the empirical autocorrelations of squared residuals. The McLeod-Li test is known to respond well to autoregressive conditional heteroskedasticity $(\mathrm{ARCH})$ but tends to lack power against many other interesting types of nonlinearity that do not have apparent ARCH structures.

In addition to tests based on the empirical autocorrelations of the second or higher power of residuals, we also investigate tests that involve empirical cross-correlations between residuals and their squares (or, more generally, cross-correlations between different powers of the residuals). Lawrance and Lewis $(1985,1987)$ put forward the idea of using such cross-correlations to identify nonlinear dependence and examined analytically the cross-correlation functions for certain types of nonlinear models. Their analysis, however, focused only on visual inspection of individual cross-correlations and they did not consider the effects of parameter estimation.

In what follows we tackle these problems by developing portmanteau tests based on the generalized correlations of residuals from linear models. The proposed tests are easy to implement and have chi-square asymptotic null distributions under general regularity conditions. Furthermore, tests based on cross-correlations are shown to be more powerful against many types of nonlinearity compared to the familiar test based on squared-residual autocorrelations.

The paper is organized as follows. In Section 2 we discuss residual-based generalized correlations and the associated portmanteau tests for linearity, and present some relevant asymptotic results. Section 3 examines the finite-sample properties of the proposed tests by means of Monte Carlo experiments. Section 4 presents an application to time series of stock returns. Section 5 summarizes and concludes. 


\section{Generalized Correlations And PORTMANTEAU STATISTICS}

Consider a second-order stationary, short-range dependent, real-valued stochastic process $\left\{X_{t}\right\}$ with mean $\mu$ satisfying

$$
X_{t}-\mu=\Psi(L) \varepsilon_{t}, \quad t \in \mathbb{Z}
$$

where

$$
\Psi(z)=1+\sum_{j=1}^{\infty} \psi_{j}(\boldsymbol{\delta}) z^{j}, \quad z \in \mathbb{C},
$$

$\left\{\psi_{j}(\boldsymbol{\delta})\right\}$ is an absolutely summable sequence of weights, assumed to be known functions of a finite-dimensional (row) vector $\delta$ of unknown parameters, $\left\{\varepsilon_{t}\right\}$ is strictly stationary white noise, and $L$ denotes the lag operator. A leading example of a parametric model which gives rise to a process that is representable as in (1) is the autoregressive moving average (ARMA) model. In this case, the transfer function $\Psi(z)$ is of the form

$$
\Psi(z)=B(z) / A(z), \quad z \in \mathbb{C},
$$

where, for some fixed $p, q \in \mathbb{N} \cup\{0\}$ such that $p+q>0, A(z)=1-\sum_{i=1}^{p} \alpha_{i} z^{i}$, with $A(z) \neq 0$ for all $|z| \leqslant 1, B(z)=1+\sum_{i=1}^{q} \beta_{i} z^{i}$, and $\delta=\left(\alpha_{1}, \ldots, \alpha_{p}, \beta_{1}, \ldots, \beta_{q}\right)$.

A stochastic process $\left\{X_{t}\right\}$ is typically characterized as linear if it admits the moving-average (MA) representation (1) with $\left\{\varepsilon_{t}\right\}$ being independent and identically distributed (i.i.d.) random variables. This is the notion of linearity considered by McLeod and Li (1983), Lawrance and Lewis (1985, 1987), Bickel and Bühlmann (1996), Berg, Paparoditis, and Politis (2010), and Giannerini, Maasoumi, and Dagum (2015), among many others, and is the one adopted in this paper. $^{3}$ It is worth noting, however, that this is not the only characterization of linearity found in the literature. Hannan (1973), for instance, considers a second-order stationary process to be linear if its best one-step-ahead linear predictor is the best predictor (both in the meansquare sense), which is equivalent to $\left\{\varepsilon_{t}\right\}$ in (1) being a square-integrable martingale-difference sequence relative to its natural filtration. This alternative characterization of linearity does not lend itself to the type of statistical tests considered in the sequel. A test for linearity of the best predictor is discussed in Terdik and Máth (1998).

The focus of attention here are the generalized correlations of the noise $\left\{\varepsilon_{t}\right\}$ in (1). For $r, s \in \mathbb{N}$ such that $\mathrm{E}\left(\left|\varepsilon_{0}\right|^{r+s}\right)<\infty$, we define the generalized correlations of $\left\{\varepsilon_{t}\right\}$ at lag $k$ as

$$
\rho_{r s}(k)=\left\{\gamma_{r r}(0) \gamma_{s s}(0)\right\}^{-1 / 2} \gamma_{r s}(k), \quad k \in \mathbb{Z},
$$

\footnotetext{
${ }^{3}$ For example, a causal ARMA process satisfying (1)-(2) is considered to be linear if $\left\{\varepsilon_{t}\right\}$ are i.i.d. but nonlinear if $\left\{\varepsilon_{t}\right\}$ form an uncorrelated but not independent sequence (e.g., an infinite-order ARCH sequence with $\varepsilon_{t}=\eta_{t}\left(a_{0}+\right.$ $\left.\sum_{j=1}^{\infty} a_{j} \varepsilon_{t-j}^{2}\right)^{1 / 2}$ and $\mathrm{E}\left(\eta_{0}^{2}\right) \sum_{j=1}^{\infty} a_{j}<1,\left\{\eta_{t}\right\}$ being i.i.d. zero-mean random variables).
} 
where $\gamma_{r s}(k)=\operatorname{Cov}\left(\varepsilon_{0}^{r}, \varepsilon_{k}^{s}\right)$. Thus, (3) gives the autocorrelations of $\left\{\varepsilon_{t}\right\}$ for $r=s=1$, the autocorrelations of $\left\{\varepsilon_{t}^{2}\right\}$ for $r=s=2$, and cross-correlations of the type considered by Lawrance and Lewis $(1985,1987)$ for $(r, s) \in\{(1,2),(2,1)\}$. Linearity of $\left\{X_{t}\right\}$ implies that $\rho_{r s}(k)=0$ for all $k \neq 0$.

When an estimator $\hat{\boldsymbol{\theta}}=(\hat{\mu}, \hat{\boldsymbol{\delta}})$ of $\boldsymbol{\theta}=(\mu, \boldsymbol{\delta})$ is available, one may use residuals $\left\{\hat{\varepsilon}_{t} ; t=\right.$ $1,2, \ldots, T\}$ (to be defined in a precise manner later) in place of the unobservable noise $\left\{\varepsilon_{t}\right\}$. For $r, s \in \mathbb{N}$, we define the empirical generalized correlations of the residuals at lag $k$ as

$$
\hat{\rho}_{r s}(k)=\left\{\hat{\gamma}_{r r}(0) \hat{\gamma}_{s s}(0)\right\}^{-1 / 2} \hat{\gamma}_{r s}(k), \quad k=0, \pm 1, \ldots, \pm(T-1),
$$

where $\hat{\gamma}_{r s}(k)=T^{-1} \sum_{t=1}^{T-k} f_{r}\left(\hat{\varepsilon}_{t}\right) f_{s}\left(\hat{\varepsilon}_{t+k}\right)$ for $k \geqslant 0, \hat{\gamma}_{r s}(k)=\hat{\gamma}_{s r}(-k)$ for $k<0$, and $f_{b}\left(\xi_{t}\right)=$ $\xi_{t}^{b}-T^{-1}\left(\xi_{1}^{b}+\cdots+\xi_{T}^{b}\right)$ for any collection of random variables $\left\{\xi_{t}\right\}$ and $b \in \mathbb{N}$. Tests for linearity of $\left\{X_{t}\right\}$ may then be based on portmanteau test statistics of the form

$$
\stackrel{\circ}{Q s}_{r}(m)=T \sum_{k=1}^{m} \hat{\rho}_{r s}^{2}(k)
$$

for some $r, s, m \in \mathbb{N}$ such that $r+s>2$ and $m<T$.

In order to develop asymptotic distribution theory for residual-based generalized correlations and associated portmanteau tests, the following assumptions are made (in the sequel, limits in stochastic-order symbols are taken by letting $T \rightarrow \infty)$ :

A1: $\left\{\varepsilon_{t}\right\}$ are i.i.d. with $\mathrm{E}\left(\varepsilon_{0}\right)=0$ and $0<\mathrm{E}\left(\varepsilon_{0}^{2}\right)<\infty$.

A2: $\Psi(z)$ is holomorphic in an open neighbourhood of the closed disc $|z| \leqslant 1$, does not vanish at any $|z| \leqslant 1$, and is differentiable with respect to $\delta$.

A3: $\hat{\boldsymbol{\theta}}-\boldsymbol{\theta}=O_{p}\left(T^{-1 / 2}\right)$.

A4: $\partial \tilde{\gamma}_{r s}(k) / \partial \boldsymbol{\theta}=O_{p}\left(T^{-1 / 2}\right)$ for $k \in\{0,1, \ldots, T-1\}$ and $r, s \in \mathbb{N}$ such that $r+s>2$ and $\mathrm{E}\left[\left|\varepsilon_{0}\right|^{2(r+s)}\right]<\infty$, where $\tilde{\gamma}_{r s}(k)=T^{-1} \sum_{t=1}^{T-k} f_{r}\left(\varepsilon_{t}\right) f_{s}\left(\varepsilon_{t+k}\right)$.

Assumption A1 amounts to linearity of $\left\{X_{t}\right\}$ in our setting. Under A2, $1 / \Psi(z)$ has the convergent power series expansion $1 / \Psi(z)=\phi_{0}(\boldsymbol{\delta})-\sum_{j=1}^{\infty} \phi_{j}(\boldsymbol{\delta}) z^{j}$ for $|z| \leqslant 1$, with $\phi_{0}(\boldsymbol{\delta})=1$ and

$$
\phi_{j}(\boldsymbol{\delta})=\psi_{j}(\boldsymbol{\delta})-\sum_{i=1}^{j-1} \phi_{j-i}(\boldsymbol{\delta}) \psi_{i}(\boldsymbol{\delta}), \quad j \in \mathbb{N},
$$

and, consequently, $\left\{X_{t}\right\}$ admits the autoregressive (AR) representation

$$
X_{t}-\mu=\sum_{j=1}^{\infty} \phi_{j}(\boldsymbol{\delta})\left(X_{t-j}-\mu\right)+\varepsilon_{t}, \quad t \in \mathbb{Z} .
$$

Hence, given an estimator $\hat{\boldsymbol{\theta}}$ based on a finite stretch $\left(X_{0}, X_{1}, \ldots, X_{T}\right)$ of $\left\{X_{t}\right\}$, residuals may 
be defined as (cf. Kreiss (1991))

$$
\hat{\varepsilon}_{t}=X_{t}-\hat{\mu}-\sum_{j=1}^{t} \phi_{j}(\hat{\boldsymbol{\delta}})\left(X_{t-j}-\hat{\mu}\right), \quad t=1,2, \ldots, T .
$$

Estimators of $\boldsymbol{\theta}$ satisfying assumption A3 may be obtained by quasi-maximum likelihood or instrumental-variables methods under suitable regularity conditions (see, e.g., Hannan (1973); Dunsmuir (1979); Hosoya and Taniguchi (1982); Kuersteiner (2001)). In the ARMA case specified by (2), assumptions A2-A4 hold true, under an i.i.d. assumption about $\left\{\varepsilon_{t}\right\}$, as long as the polynomials $A(z)$ and $B(z)$ have no zeros in common and $A(z) B(z) \neq 0$ for all $|z| \leqslant 1$.

We have the following result for the asymptotic distribution of a finite set of empirical generalized correlations of the residuals defined by (4) under the assumption that $\left\{X_{t}\right\}$ is linear.

Theorem 1 Suppose that $\left\{X_{t}\right\}$ satisfies (1) and assumptions A1-A4 hold. Then, for any fixed $m \in \mathbb{N}$ and $r, s \in \mathbb{N}$ such that $r+s>2$ and $\mathrm{E}\left[\left|\varepsilon_{0}\right|^{2(r+s)}\right]<\infty$, the asymptotic distribution of $\sqrt{T}\left(\hat{\rho}_{r s}(1), \ldots, \hat{\rho}_{r s}(m)\right)$, as $T \rightarrow \infty$, is Gaussian with zero mean vector and identity covariance matrix.

Proof: For a fixed $m<T$, a Taylor expansion of $\hat{\gamma}_{r s}(k)$ about $\boldsymbol{\theta}$ leads to

$$
\hat{\gamma}_{r s}(k)=\tilde{\gamma}_{r s}(k)+\frac{\partial \tilde{\gamma}_{r s}(k)}{\partial \boldsymbol{\theta}}(\hat{\boldsymbol{\theta}}-\boldsymbol{\theta})^{\prime}+O_{p}\left(T^{-1}\right)=\tilde{\gamma}_{r s}(k)+O_{p}\left(T^{-1}\right), \quad k=0,1, \ldots, m .
$$

Hence, the distribution of $\sqrt{T}\left(\hat{\gamma}_{r s}(1)-\gamma_{r s}(1), \ldots, \hat{\gamma}_{r s}(m)-\gamma_{r s}(m)\right)$ is asymptotically the same as the distribution of $\sqrt{T}\left(\tilde{\gamma}_{r s}(1)-\gamma_{r s}(1), \ldots, \tilde{\gamma}_{r s}(m)-\gamma_{r s}(m)\right)$. Furthermore, putting $\dot{f}_{b}\left(\varepsilon_{t}\right)=$ $\varepsilon_{t}^{b}-\mathrm{E}\left(\varepsilon_{0}^{b}\right), b \in \mathbb{N}$, and noting that $T^{-1} \sum_{t=1}^{T} \dot{f}_{b}\left(\varepsilon_{t}\right)=O_{p}\left(T^{-1 / 2}\right)$ for $b \in\{r, s\}$, it is not difficult to show that $\tilde{\gamma}_{r s}(k)-T^{-1} \sum_{t=1}^{T} \dot{f}_{r}\left(\varepsilon_{t}\right) \dot{f}_{s}\left(\varepsilon_{t+k}\right)=o_{p}\left(T^{-1 / 2}\right)$ for $0 \leqslant k \leqslant m$. Therefore, recalling that $\gamma_{r s}(k)=0$ for all $k \neq 0$ under assumption $\mathrm{A} 1$, by an application of the central limit theorem for strictly stationary, finitely dependent sequences (e.g., Anderson (1971, Theorem 7.7.6)) to the normalized partial sum $T^{-1 / 2} \sum_{t=1}^{T}\left(\dot{f}_{r}\left(\varepsilon_{t}\right) \dot{f}_{s}\left(\varepsilon_{t+1}\right), \ldots, \dot{f}_{r}\left(\varepsilon_{t}\right) \dot{f}_{s}\left(\varepsilon_{t+m}\right)\right)$ we may conclude that, as $T \rightarrow \infty$, the distribution of $\sqrt{T}\left\{\gamma_{r r}(0) \gamma_{s s}(0)\right\}^{-1 / 2}\left(\hat{\gamma}_{r s}(1), \ldots, \hat{\gamma}_{r s}(m)\right)$ converges weakly to the standard normal distribution on $\mathbb{R}^{m}$. The assertion of the theorem follows from this result and the fact that $\hat{\gamma}_{b b}(0)=\tilde{\gamma}_{b b}(0)+O_{p}\left(T^{-1}\right)=\gamma_{b b}(0)+o_{p}(1)$ for $b \in\{r, s\}$.

We note that, for $r=s=2$ and $\Psi(z)$ specified as in (2), the central limit theorem of McLeod and $\mathrm{Li}$ (1983) is retrieved from Theorem 1. We also note that, as in McLeod and Li (1983), instead of using $\dot{Q}_{r s}(m)$ to test for linearity of $\left\{X_{t}\right\}$, asymptotically equivalent statistics of the form

$$
Q_{r s}(m)=T(T+2) \sum_{k=1}^{m}(T-k)^{-1} \hat{\rho}_{r s}^{2}(k),
$$

may be considered (with $r+s>2$ ), which are similar in spirit to the popular modification of the Box-Pierce statistic $Q_{11}(m)$ proposed by Ljung and Box (1978). It is readily seen that, under 
the conditions of Theorem 1, the asymptotic distribution of the statistics ${ }_{Q}{ }_{r s}(m)$ and $Q_{r s}(m)$ is chi-square with $m$ degrees of freedom. The implementation of tests based on statistics such as $\dot{Q}_{r s}(m)$ and $Q_{r s}(m)$ is straightforward and computationally inexpensive. ${ }^{4}$

\section{Monte Carlo Simulations}

This section presents simulation results regarding the properties of portmanteau tests for linearity. In addition to the finite-sample size and power properties of the tests, we also examine the effects of non-Gaussian noise, measurement errors, correlation order, and multiple testing.

\subsection{Simulation Design}

The following data-generating processes (DGPs) are used in the simulations:

M1: $X_{t}=0.8 X_{t-1}+\varepsilon_{t}$

M2: $X_{t}=0.6 X_{t-1}-0.5 X_{t-2}+\varepsilon_{t}$

M3: $X_{t}=0.8 \varepsilon_{t-1}+\varepsilon_{t}$

M4: $X_{t}=0.8 X_{t-1}+0.15 X_{t-2}+0.3 \varepsilon_{t-1}+\varepsilon_{t}$

M5: $X_{t}=0.6 X_{t-1}+0.4 \varepsilon_{t-1}+\varepsilon_{t}$

M6: $X_{t}=0.8 X_{t-1} I\left(X_{t-1} \leqslant-1\right)-0.8 X_{t-1} I\left(X_{t-1}>-1\right)+\varepsilon_{t}$

M7: $X_{t}=-0.5 X_{t-1} I\left(X_{t-1} \leqslant 1\right)+0.4 X_{t-1} I\left(X_{t-1}>1\right)+\varepsilon_{t}$

M8: $X_{t}=-0.5 X_{t-1}\left\{1-G\left(X_{t-1}\right)\right\}+0.4 X_{t-1} G\left(X_{t-1}\right)+\varepsilon_{t}$

M9: $X_{t}=0.8 X_{t-1}\left\{1-G\left(X_{t-1}\right)\right\}-0.8 X_{t-1} G\left(X_{t-1}\right)+\varepsilon_{t}$

M10: $X_{t}=0.8\left|X_{t-1}\right|^{1 / 2}+\varepsilon_{t}$

M11: $X_{t}=Y_{t}^{2}+\varepsilon_{t}, \quad Y_{t}=0.6 Y_{t-1}+\nu_{t}$

M12: $X_{t}=\sigma_{t} \varepsilon_{t}, \quad \sigma_{t}^{2}=0.1+0.6 X_{t-1}^{2}$

M13: $X_{t}=\sigma_{t} \varepsilon_{t}, \quad \sigma_{t}^{2}=0.01+0.12 X_{t-1}^{2}+0.85 \sigma_{t-1}^{2}$

M14: $X_{t}=\sigma_{t} \varepsilon_{t}, \quad \ln \sigma_{t}^{2}=0.01+0.3\left\{\left|\varepsilon_{t-1}\right|-\mathrm{E}\left(\left|\varepsilon_{t-1}\right|\right)\right\}-0.8 \varepsilon_{t-1}+0.9 \ln \sigma_{t-1}^{2}$

M15: $X_{t}=0.4 X_{t-1}-0.3 X_{t-2}+\left(0.8+0.5 X_{t-1}\right) \varepsilon_{t-1}+\varepsilon_{t}$

M16: $X_{t}=0.5-\left(0.4-0.4 \varepsilon_{t-1}\right) X_{t-1}+\varepsilon_{t}$

\footnotetext{
${ }^{4}$ For example, the full set of Monte Carlo experiments reported in Section 3 took approximately 3 hours to carry out in MATLAB running under Windows 7 (64-bit) on a laptop with Intel Core i7 $2.60 \mathrm{GHz}$ processor and $8 \mathrm{~GB}$ of RAM.
} 
M17: $X_{t}=0.8 \varepsilon_{t-2}^{2}+\varepsilon_{t}$

M18: $X_{t}=-0.3 \varepsilon_{t-1}+\left(0.2+0.4 \varepsilon_{t-1}-0.25 \varepsilon_{t-2}\right) \varepsilon_{t-2}+\varepsilon_{t}$

Unless stated otherwise, $\left\{\varepsilon_{t}\right\}$ and $\left\{\nu_{t}\right\}$ are i.i.d. standard normal random variables independent of each other, $G(x)=1 /\left(1+e^{-x}\right)$ is the logistic distribution function, and $I(A)$ denotes the indicator of event $A$. The DGPs cover a variety of linear and nonlinear processes used in the literature, namely ARMA [M1-M5], threshold AR (TAR) [M6, M7], smooth-transition AR (STAR) [M8, M9], fractional AR (FAR) [M10], square AR (SQAR) [M11], ARCH [M12], generalized ARCH (GARCH) [M13], exponential GARCH (EGARCH) [M14], bilinear (BL) [M15, M16], and nonlinear MA (NLMA) [M17, M18]. ${ }^{5}$

In the experiments, 5000 independent artificial time series $\left\{X_{t}\right\}$ of length $100+T$, with $T \in$ $\{200,500,1000\}$, are generated according to M1-M18, but only the last $T$ data points of each series are used to carry out portmanteau tests for linearity. As preliminary analysis indicated that, for relatively short time series, tests based on the statistics $Q_{r s}(m)$ defined in (7) control the Type I error probability somewhat more successfully (albeit marginally) than tests based on the statistics $\stackrel{\circ}{r s}_{r}(m)$ defined in (5), we shall henceforth focus on the former.

Unless indicated otherwise, the tests are applied to least-squares residuals from an AR model for $\left\{X_{t}\right\}$ the order of which is determined by the Bayesian information criterion (BIC). The $\mathrm{BIC}$ is defined according to Method 1 of $\mathrm{Ng}$ and Perron (2005) with the maximum allowable order set equal to $\left\langle 8(T / 100)^{1 / 4}\right\rangle$, where $\langle x\rangle$ denotes the greatest integer in $x .^{6}$ Employing an AR model with data-dependent order as the null specification is not only computationally convenient but also theoretically attractive. Even when the DGP is not a finite-order AR process, an AR model the order of which increases with the sample size may be viewed as a finiteparameter approximation to a linear process that admits the infinite-order AR representation (6). If the order of the AR approximation grows at a suitable rate, the approximation error becomes small as $T$ increases, and estimates of the parameters in (6) obtained from the approximating autoregression are consistent and asymptotically normal (see Berk (1974); Bhansali (1978); Lewis and Reinsel (1985)).

\subsection{EMPIRICAL SIZE AND POWER}

The Monte Carlo rejection frequencies of tests, of nominal level 0.05 , based on the statistics $Q_{12}(m), Q_{21}(m)$ and $Q_{22}(m)$, with $m \in\{1,2, \ldots,\langle\sqrt{T}\rangle\}$, are shown in Figures $1-3 .{ }^{7}$ Under

\footnotetext{
${ }^{5}$ The DGPs are taken from Lee, White, and Granger (1993) [M11, M15, M18], Barnett, Gallant, Hinich, Jungeilges, Kaplan, and Jensen (1997) [M4], Hong and Lee (2003) [M2, M14], Hong and White (2005) [M10], and Giannerini, Maasoumi, and Dagum (2015) [M1, M3, M5, M6, M7, M12, M13, M16, M17]; M8 and M9 are smooth-transition variants of $M 7$ and $M 6$, respectively.

${ }^{6}$ Very similar results are obtained using Akaike's information criterion instead of the BIC.

${ }^{7}$ Simulation results for tests of nominal level 0.01 and 0.10 are not reported, due to space constraints, but are available upon request.
} 
linear DGPs (M1-M5), all three portmanteau tests have empirical levels which do not differ significantly from the nominal level regardless of the sample size $T$ and the number of generalized correlations $m$ used to construct the test statistic. It is noteworthy that the tests work well in the case of linear DGPs which do not have a finite-order AR structure (M3-M5), suggesting that $A R$ approximations provide a useful way of modelling dynamics under the null hypothesis in this context.

For all but two of the nonlinear DGPs (M6-M18), at least one of the two cross-correlation tests $Q_{12}$ and $Q_{21}$ has higher rejection frequencies than the $Q_{22}$ test, especially when $T \leqslant 500$. The test based on $Q_{22}$ has a clear advantage in the case of time series generated according to M12 and $\mathrm{M} 13$, which is not perhaps surprising since $Q_{22}$ is asymptotically equivalent to a Lagrange multiplier statistic for testing linearity against $\mathrm{ARCH}$ (see Luukkonen, Saikkonen, and Teräsvirta (1988)). The power of all the tests generally improves as $T$ increases.

\subsection{NON-GAUSSIAN NOISE}

To investigate the sensitivity of the simulation results with respect to non-Gaussianity of the noise in the DGP, we consider artificial time series (of length $T=500$ ) generated accorded M1-M18 with $\varepsilon_{t}$ having either Student's $t$ distribution with $d$ degrees of freedom or a gamma distribution with shape parameter $d$ and scale parameter 1 . (The distributions are recentred and/or rescaled so as to have zero mean and unit variance). We take $d \in\{10,11, \ldots, 19,20\}$, a range of values which is sufficiently representative of some of the distributional characteristics (e.g., mild asymmetry and leptokurtosis) of many economic and financial time series. Following the suggestion in Tong (1990, p. 324) that, when constructing tests for uncorrelatedness, autocorrelations at low lags should be watched more closely than autocorrelations at high lags, we set $m=\langle\ln T\rangle$ (see also Tsay (2010, p. 33)).

For the sake of expositional simplicity and space conservation, the rejection frequencies of tests (of nominal level 0.05) are averaged over the linear (M1-M5) and nonlinear (M6-M18) DGPs, and are shown in Figure 4 (straight lines indicate the average rejection frequencies of tests under Gaussian noise). The results indicate that the level and power properties of the tests are generally insensitive with respect to the value of the parameter $d$. In the case of gamma distributed noise, $Q_{22}\left(Q_{21}\right)$ has marginally lower (higher) average power compared to the Gaussian case.

\subsection{MeAsurement ERRORS}

Economic and financial data are often contaminated by measurement errors due to, inter alia, sampling, self-reporting or imperfect data sources. To investigate the potential effect of such measurement errors on tests for nonlinearity, we consider contaminated series (of length 
$T=500)$ generated according to $X_{t}^{*}=X_{t}+\sigma_{\eta} \eta_{t}$, where $X_{t}$ comes from M1-M18 and $\left\{\eta_{t}\right\}$ are i.i.d. random variables, independent of $\left\{\varepsilon_{t}\right\}$ and $\left\{\nu_{t}\right\}$, having either Student's $t$ distribution with 10 degrees of freedom or a gamma distribution with shape parameter 10 and scale parameter 1 (recentred and/or rescaled to have zero mean and unit variance). The variance of the measurement error is allowed to be proportional to the sample variance $\hat{\sigma}_{x}^{2}$ of $\left(X_{1}, \ldots, X_{T}\right)$, that is $\sigma_{\eta}^{2}=\omega^{2} \hat{\sigma}_{x}^{2}$, where $\omega^{2} \in\{0.005,0.010, \ldots, 0.060,0.065\}$. The range of values for the noise-tosignal ratio $\omega$ is calibrated according to Koreisha and Fang (1999) and allows for up to $25 \%$ contamination by measurement errors.

Tests for linearity based on $Q_{r s}(m)$, with $r, s \in\{1,2\}$ and $m=\langle\ln T\rangle$, are implemented as described in Section 3.1 using $\left\{X_{t}^{*}\right\}$ in place of $\left\{X_{t}\right\}$. The rejection frequencies of tests (of nominal level 0.05), averaged across the linear (M1-M5) and nonlinear (M6-M18) DGPs, are shown in Figure 5. The tests exhibit no substantial size distortion, regardless of the contamination rate and the distribution of the noise. Some power loss is observed as the contamination rate increases, but the reduction in power is not of the magnitude that makes the tests unattractive for applications.

\subsection{Higher-Order CORRELATIONS}

Although the discussion in much of the paper focuses on tests with $r, s \in\{1,2\}$, the use of higher values for $(r, s)$ is, of course, possible. To examine whether power gains may be made by using higher-order generalized correlations, we compute the empirical power of tests based on $Q_{r s}(m)$ with $r, s \in\{1,2, \ldots, 6\}$ and $m=\langle\ln T\rangle$. The rejection frequencies of tests (of nominal level 0.05 ) for $T=500$, averaged across the nonlinear DGPs (M6-M18), are reported in Table 1. The results indicate that there are generally no power improvements associated with the use of higher-order generalized correlations; for instance, tests based on $Q_{12}$ and $Q_{32}$ have almost the same (average) rejection frequencies. Furthermore, it is worth bearing in mind that the asymptotic justification of portmanteau tests associated with high values of $(r, s)$ requires finiteness of a fairly large number of moments (cf. Theorem 1). This requirement may be at odds with the characteristics of many economic and financial time series (e.g., equity returns, exchange rate returns, interest rates), for which it is often argued that they only possess unconditional moments of relatively low order (see, e.g., Koedijk, Schafgans, and de Vries (1990); Jansen and de Vries (1991); de Lima (1997)).

\subsection{Multiple Testing}

In practice, linearity is often tested using several tests (e.g., $Q_{r s}(m), r, s \in\{1,2\}$ ) jointly and/or several values of $m$. However, unless adjustments for multiple testing are made, there is an increased risk of overstating the significance of nonlinearity when many tests are carried out using the same set of data (see Psaradakis (2000)). This is due to the fact that, if the linearity 
hypothesis is rejected when at least one of the tests leads to a rejection, the overall Type I error probability associated with the multiple testing procedure (i.e., the probability of at least one erroneous rejection) can be well in excess of the nominal level of each individual test.

A simple Bonferroni-type adjustment for multiple testing based on Simes (1986) is considered here, which may be implemented as follows. Let $P_{(1)} \leqslant P_{(2)} \leqslant \cdots \leqslant P_{(N)}$ denote the ordered (asymptotic) $P$-values associated with the set of portmanteau test statistics under consideration. Multiplicity-adjusted $P$-values are then calculated as $\tilde{P}_{(i)}=\min \left\{N P_{(i)} / i, 1\right\}$, $i \in\{1,2, \ldots, N\}$, and the null hypothesis of linearity is rejected at overall level $\alpha \in(0,1)$ if $\min _{1 \leqslant i \leqslant N} \tilde{P}_{(i)} \leqslant \alpha$. Simes' procedure is generally less conservative than the classical Bonferroni or Šidák procedures, especially when several highly correlated test statistics are involved. ${ }^{8}$

In Table 2 we report Monte Carlo estimates of the probability that at least one of the tests under consideration rejects the null hypothesis of linearity at the 0.05 level (when $T=500$ ). Case A refers to the situation when linearity is tested using the statistics $Q_{12}(m), Q_{21}(m)$ and $Q_{22}(m)$ with $m=\langle\ln T\rangle=6(N=3)$; in Case B linearity is tested using $Q_{12}(m), Q_{21}(m), Q_{22}(m)$ and six different values of $m$, namely $m \in\{1, \ldots, 6\}(N=18)$. The advantage of adjusting for multiplicity in testing is immediately evident. Using unadjusted $P$-values, the probability that one or more of the tests will erroneously reject the null hypothesis under M1-M5 ranges from 0.14 to 0.29 . By contrast, the multiple testing procedures generally have an overall Type I error probability that is quite close to the nominal 0.05 level (in spite of the fact that they do not account for the dependence structure of the individual test statistics). Moreover, the protection against an excessive overall Type I error probability is not achieved at the cost of a systematic loss of the ability of the tests to reject correctly the linearity hypothesis under M6-M18.

\section{EMpirical Application}

In this section portmanteau tests for linearity are applied to a set of weekly stock returns, spanning the period 1993-2007 (781 observations), for 100 companies from the Standard \& Poor's 500 Composite index. The selected series are part of the data set analyzed by Kapetanios (2009) and are such that the hypothesis of strict stationarity cannot be rejected for any of them (at $5 \%$ significance level). The presence of nonlinearity in asset returns has important implications for, inter alia, pricing, risk management, and forecasting.

As in Section 3, we test for neglected nonlinearity in an AR model for each time series, the order of which is determined by minimizing the BIC over the range $\left\{0,1, \ldots,\left\langle 8(T / 100)^{1 / 4}\right\rangle\right\}$. The asymptotic $P$-values for tests based on $Q_{12}(m), Q_{21}(m)$ and $Q_{22}(m)$, with $m=\langle\ln T\rangle$, are reported in Table 5. In order to guard against the danger of overstating the significance of nonlinearity because of the use of three different tests, we also report the $P$-values of the

\footnotetext{
${ }^{8}$ It also yields the same critical values as the multiple testing procedure of Benjamini and Hochberg (1995) that controls the so-called false discovery rate (i.e., the expected proportion of erroneous rejections over all rejections).
} 
individual test statistics adjusted for multiplicity using the method described in Section 3.6.

Using unadjusted test $P$-values, evidence against linearity is found in 82 stock returns (at $5 \%$ significance level) on the basis of the $Q_{22}$ test. This arguably is not a very surprising finding since conditional heteroskedasticity is a characteristic feature of many asset returns. Linearity is also rejected by at least one of the cross-correlation $Q_{12} / Q_{21}$ tests in 66 cases. Using multiplicity-adjusted $P$-values, evidence against linearity is found by at least one of the three tests in $85 \%$ of stock returns (at $5 \%$ significance level). We conclude, therefore, that the vast majority of the stock returns considered in our analysis exhibit nonlinear features which cannot be captured by a linear model with i.i.d. noise.

\section{SUMMARY}

This paper considered portmanteau tests for linearity of stationary time series based on generalized correlations of residuals. The proposed tests are easy to implement, have a chi-square large-sample null distribution, and good size and power properties in finite samples. The simulation results indicated that the cross-correlation tests $Q_{12}$ and $Q_{21}$ are useful in identifying various types of nonlinearity and are generally more powerful than the popular $Q_{22}$ test based on squared-residual autocorrelations. An application to time series of stock returns illustrated the practical use of the tests. 


\section{References}

Anderson, T. W. (1971): The Statistical Analysis of Time Series. Wiley, New York.

Barnett, W. A., A. R. Gallant, M. J. Hinich, J. A. Jungeilges, D. T. Kaplan, and M. J. JENSEN (1997): "A single-blind controlled competition among tests for nonlinearity and chaos," Journal of Econometrics, 82, 157-192.

Benjamini, Y., And Y. Hochberg (1995): "Controlling the false discovery rate: a practical and powerful approach to multiple testing," Journal of the Royal Statistical Society B, 57, 289-300.

Berg, A., E. Paparoditis, and D. N. Politis (2010): "A bootstrap test for time series linearity," Journal of Statistical Planning and Inference, 140, 3841-3857.

BERK, K. N. (1974): "Consistent autoregressive spectral estimates," Annals of Statistics, 2, 489-502.

BHANSALI, R. J. (1978): "Linear prediction by autoregressive model fitting in the time domain," Annals of Statistics, 6, 224-231.

Bickel, P. J., And P. Bühlmann (1996): "What is a linear process?," Proceedings of the National Academy of Sciences of the USA, 93, 12128-12131.

DE LIMA, P. J. F. (1997): "On the robustness of nonlinearity tests to moment condition failure," Journal of Econometrics, 76, 251-280.

DUNSMUIR, W. (1979): "A central limit theorem for parameter estimation in stationary vector time series and its application to models for a signal observed with noise," Annals of Statistics, 7, 490-506.

Giannerini, S., E. MaAsoumi, and E. DaGum (2015): "Entropy testing for nonlinear serial dependence in time series," Biometrika, 102, 661-675.

HANNAN, E. J. (1973): "The asymptotic theory of linear time-series models," Journal of Applied Probability, 10, 130-145.

HONG, Y., AND T.-H. LEE (2003): "Diagnostic checking for the adequacy of nonlinear time series models," Econometric Theory, 19, 1065-1121.

HONG, Y., AND H. WHITE (2005): "Asymptotic distribution theory for nonparametric entropy measures of serial dependence," Econometrica, 73, 837-901.

Hosoya, Y., AND M. TANIGUCHI (1982): "A central limit theorem for stationary processes and the parameter estimation of linear processes," Annals of Statistics, 10, 132-153.

JANSEN, D. W., AND C. G. DE VRIES (1991): "On the frequency of large stock returns: putting booms and busts into perspective," Review of Economics and Statistics, 73, 18-24. 
KAPETANIOS, G. (2009): "Testing for strict stationarity in financial variables," Journal of Banking and Finance, 33, 2346-2362.

KoediJK, K., M. Schafgans, and C. DE VRIES (1990): "The tail index of exchange rate returns," Journal of International Economics, 29, 93-108.

KoREISHA, S. G., AND Y. FANG (1999): "The impact of measurement errors on ARMA prediction," Journal of Forecasting, 18, 95-109.

KREISS, J.-P. (1991): "Estimation of the distribution function of noise in stationary processes," Metrika, 38, 285-297.

KUERSteineR, G. M. (2001): "Optimal instrumental variables estimation for ARMA models," Journal of Econometrics, 104, 359-405.

LAWRANCE, A. J., AND P. A. W. LeWIS (1985): "Modelling and residual analysis of nonlinear autoregressive time series in exponential variables," Journal of the Royal Statistical Society $B, 47,165-202$.

(1987): "Higher-order residual analysis for nonlinear time series with autoregressive correlation structures," International Statistical Review, 55, 21-35.

Lee, T.-H., H. White, and C. W. J. Granger (1993): "Testing for neglected nonlinearity in time series models: a comparison of neural network methods and alternative tests," Journal of Econometrics, 56, 269-290.

LEWIS, R., AND G. C. REINSEL (1985): "Prediction of multivariate time series by autoregressive model fitting," Journal of the Multivariate Analysis, 16, 393-411.

LJUNG, G. M., AND G. E. P. Box (1978): "On a measure of lack of fit in time series models," Biometrika, 65, 297-303.

Luukkonen, R., P. Saikkonen, and T. Teräsvirta (1988): "Testing linearity in univariate time series models," Scandinavian Journal of Statistics, 15, 161-175.

MCLEOD, A. I., AND W. K. LI (1983): "Diagnostic checking ARMA time series models using squared-residual autocorrelations," Journal of Time Series Analysis, 4, 269-273.

NG, S., And P. Perron (2005): "A note on the selection of time series models," Oxford Bulletin of Economics and Statistics, 67, 115-134.

PSARADAKIS, Z. (2000): "P-value adjustments for multiple tests for nonlinearity," Studies in Nonlinear Dynamics \& Econometrics, 4, 95-100.

SIMES, R. J. (1986): "An improved Bonferroni procedure for multiple tests of significance," Biometrika, 73, 751-754.

Teräsvirta, T., D. TJøstheim, And C. W. J. Granger (2010): Modelling Nonlinear Economic Time Series. Oxford University Press, Oxford. 
TERDIK, G., AND J. MÁTH (1998): "A new test of linearity of time series based on the bispectrum," Journal of Time Series Analysis, 19, 737-753.

Tong, H. (1990): Non-linear Time Series: A Dynamical System Approach. Oxford University Press, Oxford.

TSAY, R. S. (2010): Analysis of Financial Time Series. 3rd Edition, Wiley, New York. 
Table 1: Rejection frequencies of $Q_{r s}$ tests

\begin{tabular}{c|ccccc}
\hline \hline$w$ & $Q_{1 w}$ & $Q_{w 1}$ & $Q_{2 w}$ & $Q_{w 2}$ & $Q_{w w}$ \\
\hline 2 & 0.77 & 0.40 & 0.72 & 0.72 & 0.72 \\
3 & 0.19 & 0.24 & 0.42 & 0.76 & 0.38 \\
4 & 0.65 & 0.42 & 0.60 & 0.66 & 0.53 \\
5 & 0.24 & 0.32 & 0.36 & 0.66 & 0.32 \\
6 & 0.53 & 0.42 & 0.48 & 0.60 & 0.34 \\
\hline \hline
\end{tabular}

Table 2: Rejection frequencies under multiple testing

\begin{tabular}{c|cc|cc}
\hline \hline & \multicolumn{2}{|c|}{ Case A $(N=3)$} & \multicolumn{2}{c}{ Case B $(N=18)$} \\
\cline { 2 - 5 } Unadjusted & Adjusted & Unadjusted & Adjusted \\
\hline M1 & 0.141 & 0.048 & 0.286 & 0.042 \\
M3 & 0.136 & 0.044 & 0.287 & 0.039 \\
M4 & 0.136 & 0.048 & 0.296 & 0.041 \\
M5 & 0.137 & 0.054 & 0.284 & 0.043 \\
M6 & 0.141 & 0.055 & 0.287 & 0.042 \\
M7 & 1.000 & 1.000 & 1.000 & 1.000 \\
M8 & 1.000 & 1.000 & 1.000 & 1.000 \\
M9 & 0.964 & 0.919 & 0.997 & 0.966 \\
M10 & 0.997 & 0.992 & 1.000 & 0.997 \\
M11 & 0.723 & 0.542 & 0.931 & 0.681 \\
M12 & 0.799 & 0.703 & 0.941 & 0.788 \\
M13 & 1.000 & 0.999 & 1.000 & 0.999 \\
M14 & 0.980 & 0.965 & 0.984 & 0.944 \\
M15 & 1.000 & 1.000 & 1.000 & 1.000 \\
M16 & 1.000 & 1.000 & 1.000 & 1.000 \\
M17 & 0.940 & 1.000 & 1.000 & 1.000 \\
M18 & 0.998 & 0.894 & 0.989 & 0.920 \\
\hline
\end{tabular}


Table 3: Unadjusted and multiplicity-adjusted $P$-values

\begin{tabular}{|c|c|c|c|c|c|c|c|c|c|c|c|c|c|c|c|}
\hline \multirow[b]{2}{*}{ company } & \multicolumn{3}{|c|}{ Unadjusted $P$-values } & \multicolumn{3}{|c|}{ Adjusted $P$-values } & \multirow{2}{*}{$\begin{array}{c}\text { AR } \\
\text { order }\end{array}$} & \multirow[b]{2}{*}{ company } & \multicolumn{3}{|c|}{ Unadjusted $P$-values } & \multicolumn{3}{|c|}{ Adjusted $P$-values } & \multirow{2}{*}{$\begin{array}{c}\text { AR } \\
\text { order }\end{array}$} \\
\hline & $Q_{12}$ & $Q_{21}$ & $Q_{22}$ & $Q_{12}$ & $Q_{21}$ & $Q_{22}$ & & & $Q_{12}$ & $Q_{21}$ & $Q_{22}$ & $Q_{12}$ & $Q_{21}$ & $Q_{22}$ & \\
\hline Alcoa Inc & 0.20 & 0.00 & 0.00 & 0.20 & 0.01 & 0.00 & 1 & Danaher Corp. & 0.01 & 0.00 & 0.00 & 0.01 & 0.01 & 0.00 & 1 \\
\hline Apple Inc. & 0.01 & 0.67 & 0.45 & 0.02 & 0.67 & 0.68 & 2 & Walt Disney Co. & 0.62 & 0.05 & 0.01 & 0.62 & 0.07 & 0.04 & 1 \\
\hline Adobe Systems Inc & 0.21 & 0.01 & 0.00 & 0.21 & 0.01 & 0.00 & 1 & Dow Chemical & 0.19 & 0.06 & 0.00 & 0.19 & 0.09 & 0.00 & 1 \\
\hline Analog Devices Inc & 0.07 & 0.00 & 0.00 & 0.07 & 0.00 & 0.00 & 1 & Duke Energy & 0.00 & 0.00 & 0.00 & 0.00 & 0.00 & 0.00 & 1 \\
\hline Archer-Daniels-Midland & 0.56 & 0.99 & 0.04 & 0.85 & 0.99 & 0.11 & 1 & Ecolab Inc. & 0.00 & 0.00 & 0.00 & 0.00 & 0.00 & 0.00 & 1 \\
\hline Autodesk Inc & 0.10 & 0.19 & 0.01 & 0.15 & 0.19 & 0.02 & 1 & Equifax Inc. & 0.13 & 0.23 & 0.12 & 0.20 & 0.23 & 0.37 & 1 \\
\hline American Electric Power & 0.00 & 0.00 & 0.00 & 0.00 & 0.00 & 0.00 & 1 & Edison Int'l & 0.01 & 0.00 & 0.00 & 0.01 & 0.00 & 0.00 & 2 \\
\hline AES Corp & 0.00 & 0.00 & 0.00 & 0.00 & 0.00 & 0.00 & 1 & EMC Corp. & 0.01 & 0.00 & 0.00 & 0.01 & 0.00 & 0.00 & 1 \\
\hline AFLAC Inc & 0.00 & 0.00 & 0.00 & 0.00 & 0.00 & 0.00 & 1 & Emerson Electric & 0.31 & 0.03 & 0.00 & 0.31 & 0.04 & 0.00 & 1 \\
\hline Allergan Inc & 0.04 & 0.09 & 0.00 & 0.06 & 0.09 & 0.00 & 1 & Equity Residential & 0.45 & 0.22 & 0.00 & 0.45 & 0.34 & 0.00 & 1 \\
\hline American Intl Group Inc & 0.03 & 0.00 & 0.00 & 0.03 & 0.00 & 0.00 & 1 & EQT Corporation & 0.20 & 0.00 & 0.00 & 0.20 & 0.00 & 0.00 & 1 \\
\hline Aon plc & 0.02 & 0.00 & 0.00 & 0.02 & 0.00 & 0.00 & 1 & Eaton Corp. & 0.47 & 0.22 & 0.40 & 0.47 & 0.67 & 0.59 & 1 \\
\hline Apache Corporation & 0.37 & 0.02 & 0.00 & 0.37 & 0.03 & 0.00 & 1 & Entergy Corp. & 0.01 & 0.11 & 0.00 & 0.01 & 0.11 & 0.00 & 1 \\
\hline Anadarko Petroleum & 0.73 & 0.05 & 0.00 & 0.73 & 0.08 & 0.00 & 1 & Exelon Corp. & 0.45 & 0.43 & 0.02 & 0.45 & 0.64 & 0.05 & 1 \\
\hline Avon Products & 0.19 & 0.00 & 0.00 & 0.19 & 0.00 & 0.00 & 1 & Ford Motor & 0.01 & 0.15 & 0.00 & 0.01 & 0.15 & 0.00 & 1 \\
\hline Avery Dennison Corp & 0.00 & 0.12 & 0.00 & 0.01 & 0.12 & 0.00 & 1 & Fastenal Co & 0.28 & 0.06 & 0.03 & 0.28 & 0.09 & 0.08 & 1 \\
\hline American Express Co & 0.24 & 0.00 & 0.00 & 0.24 & 0.00 & 0.00 & 1 & Family Dollar Stores & 0.86 & 0.05 & 0.00 & 0.86 & 0.08 & 0.00 & 1 \\
\hline Bank of America Corp & 0.21 & 0.00 & 0.00 & 0.21 & 0.00 & 0.00 & 1 & FedEx Corporation & 0.04 & 0.17 & 0.00 & 0.06 & 0.17 & 0.00 & 1 \\
\hline Baxter International Inc. & 0.06 & 0.00 & 0.28 & 0.09 & 0.00 & 0.28 & 1 & Fiserv Inc & 0.01 & 0.00 & 0.00 & 0.01 & 0.00 & 0.00 & 1 \\
\hline BBT Corporation & 0.58 & 0.01 & 0.00 & 0.58 & 0.02 & 0.00 & 1 & Fifth Third Bancorp & 0.04 & 0.00 & 0.00 & 0.04 & 0.01 & 0.00 & 1 \\
\hline Best Buy Co. Inc. & 0.75 & 0.00 & 0.00 & 0.75 & 0.00 & 0.00 & 1 & Fluor Corp. & 0.00 & 0.03 & 0.12 & 0.00 & 0.04 & 0.12 & 1 \\
\hline Bard (C.R.) Inc. & 0.71 & 0.03 & 0.01 & 0.71 & 0.05 & 0.02 & 1 & Forest Laboratories & 0.03 & 0.08 & 0.64 & 0.09 & 0.12 & 0.64 & 1 \\
\hline Becton Dickinson & 0.24 & 0.07 & 0.00 & 0.24 & 0.11 & 0.00 & 1 & Frontier Commun. & 0.00 & 0.00 & 0.00 & 0.00 & 0.00 & 0.00 & 1 \\
\hline Franklin Resources & 0.38 & 0.00 & 0.00 & 0.38 & 0.00 & 0.00 & 1 & Gannett Co. & 0.64 & 0.01 & 0.00 & 0.64 & 0.01 & 0.00 & 1 \\
\hline Brown-Forman Corp & 0.29 & 0.00 & 0.04 & 0.29 & 0.00 & 0.06 & 1 & General Dynamics & 0.50 & 0.01 & 0.00 & 0.50 & 0.02 & 0.00 & 1 \\
\hline Baker Hughes Inc & 0.24 & 0.00 & 0.00 & 0.24 & 0.00 & 0.00 & 1 & General Electric & 0.00 & 0.00 & 0.00 & 0.00 & 0.00 & 0.00 & 1 \\
\hline The Bank of NY Mellon & 0.23 & 0.00 & 0.00 & 0.23 & 0.00 & 0.00 & 1 & General Mills & 0.98 & 0.87 & 0.98 & 0.98 & 1.00 & 1.00 & 1 \\
\hline Ball Corp & 0.00 & 0.00 & 0.00 & 0.00 & 0.00 & 0.00 & 1 & Genuine Parts & 0.02 & 0.02 & 0.00 & 0.02 & 0.03 & 0.00 & 1 \\
\hline Boston Scientific & 0.95 & 0.01 & 0.01 & 0.95 & 0.02 & 0.04 & 1 & Gap (The) & 0.81 & 0.00 & 0.00 & 0.81 & 0.00 & 0.00 & 1 \\
\hline
\end{tabular}




\begin{tabular}{|c|c|c|c|c|c|c|c|c|c|c|c|c|c|c|c|}
\hline Cardinal Health Inc. & 0.72 & 0.05 & 0.03 & 0.72 & 0.08 & 0.08 & 3 & Grainger Inc. & 0.37 & 0.05 & 0.00 & 0.37 & 0.07 & 0.00 & 2 \\
\hline Caterpillar Inc. & 0.08 & 0.05 & 0.01 & 0.08 & 0.07 & 0.04 & 1 & Halliburton Co. & 0.00 & 0.00 & 0.00 & 0.00 & 0.00 & 0.00 & 1 \\
\hline Chubb Corp. & 0.01 & 0.04 & 0.00 & 0.02 & 0.04 & 0.00 & 1 & Harman Int'I Ind. & 0.65 & 0.36 & 0.55 & 0.65 & 1.00 & 0.82 & 2 \\
\hline Coca-Cola Enterprises & 0.26 & 0.31 & 0.06 & 0.39 & 0.31 & 0.18 & 1 & Hasbro Inc. & 0.16 & 0.14 & 0.34 & 0.24 & 0.42 & 0.34 & 1 \\
\hline Carnival Corp. & 0.00 & 0.00 & 0.00 & 0.00 & 0.00 & 0.00 & 1 & Huntington Bancshares & 0.12 & 0.01 & 0.00 & 0.12 & 0.02 & 0.00 & 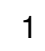 \\
\hline CIGNA Corp. & 0.24 & 0.00 & 0.70 & 0.36 & 0.00 & 0.70 & 1 & Health Care REIT & 0.98 & 0.11 & 0.00 & 0.98 & 0.16 & 0.00 & 1 \\
\hline Cincinnati Financial & 0.00 & 0.63 & 0.00 & 0.00 & 0.63 & 0.00 & 1 & Home Depot & 0.35 & 0.07 & 0.00 & 0.35 & 0.11 & 0.00 & 1 \\
\hline Clorox Co. & 0.18 & 0.00 & 0.00 & 0.18 & 0.00 & 0.00 & 1 & Hess Corporation & 0.47 & 0.28 & 1.00 & 0.71 & 0.85 & 1.00 & 1 \\
\hline Comerica Inc. & 0.39 & 0.00 & 0.01 & 0.39 & 0.00 & 0.02 & 1 & Harley-Davidson & 0.07 & 0.00 & 0.74 & 0.11 & 0.00 & 0.74 & 1 \\
\hline CMS Energy & 0.00 & 0.00 & 0.00 & 0.00 & 0.00 & 0.00 & 2 & Honeywell Int'I Inc. & 0.32 & 0.13 & 0.68 & 0.48 & 0.40 & 0.68 & 1 \\
\hline CenterPoint Energy & 0.00 & 0.00 & 0.00 & 0.00 & 0.00 & 0.00 & 1 & Hewlett-Packard & 0.16 & 0.01 & 0.00 & 0.16 & 0.01 & 0.00 & 1 \\
\hline Cabot Oil and Gas & 1.00 & 0.02 & 0.00 & 1.00 & 0.03 & 0.00 & 1 & Block $\mathrm{H}$ and $\mathrm{R}$ & 0.13 & 0.02 & 0.00 & 0.13 & 0.02 & 0.00 & 1 \\
\hline ConocoPhillips & 0.18 & 0.38 & 0.49 & 0.53 & 0.57 & 0.49 & 1 & Hormel Foods Corp. & 0.46 & 0.09 & 0.02 & 0.46 & 0.14 & 0.06 & 1 \\
\hline Campbell Soup & 0.86 & 0.00 & 0.00 & 0.86 & 0.00 & 0.00 & 1 & The Hershey Company & 1.00 & 0.20 & 0.61 & 1.00 & 0.61 & 0.91 & 1 \\
\hline CSX Corp. & 0.08 & 0.04 & 0.00 & 0.08 & 0.06 & 0.01 & 1 & Intel Corp. & 0.92 & 0.25 & 0.00 & 0.92 & 0.38 & 0.00 & 1 \\
\hline CenturyLink Inc & 0.10 & 0.00 & 0.05 & 0.10 & 0.01 & 0.08 & 1 & International Paper & 0.17 & 0.26 & 0.00 & 0.26 & 0.26 & 0.00 & 1 \\
\hline Cablevision Corp. & 0.00 & 0.00 & 0.00 & 0.00 & 0.00 & 0.00 & 1 & Interpublic Group & 0.56 & 0.00 & 0.00 & 0.56 & 0.00 & 0.00 & 1 \\
\hline Chevron Corp. & 0.04 & 0.33 & 0.01 & 0.06 & 0.33 & 0.03 & 1 & Ingersoll-Rand PLC & 0.47 & 0.05 & 0.00 & 0.47 & 0.07 & 0.00 & 1 \\
\hline Dominion Resources & 0.00 & 0.00 & 0.00 & 0.00 & 0.00 & 0.00 & 1 & Johnson Controls & 0.03 & 0.00 & 0.00 & 0.03 & 0.00 & 0.00 & 1 \\
\hline Deere and Co. & 0.86 & 0.04 & 0.00 & 0.86 & 0.07 & 0.00 & 1 & Jacobs Eng. Group & 0.13 & 0.01 & 0.20 & 0.19 & 0.02 & 0.20 & 1 \\
\hline D. R. Horton & 0.39 & 0.02 & 0.01 & 0.39 & 0.03 & 0.04 & 1 & Johnson and Johnson & 0.13 & 0.00 & 0.00 & 0.13 & 0.00 & 0.00 & 1 \\
\hline
\end{tabular}

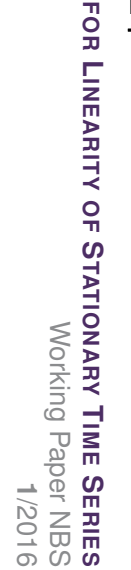


Figure 1: Rejection frequencies of $Q_{r s}$ tests: $T=200$
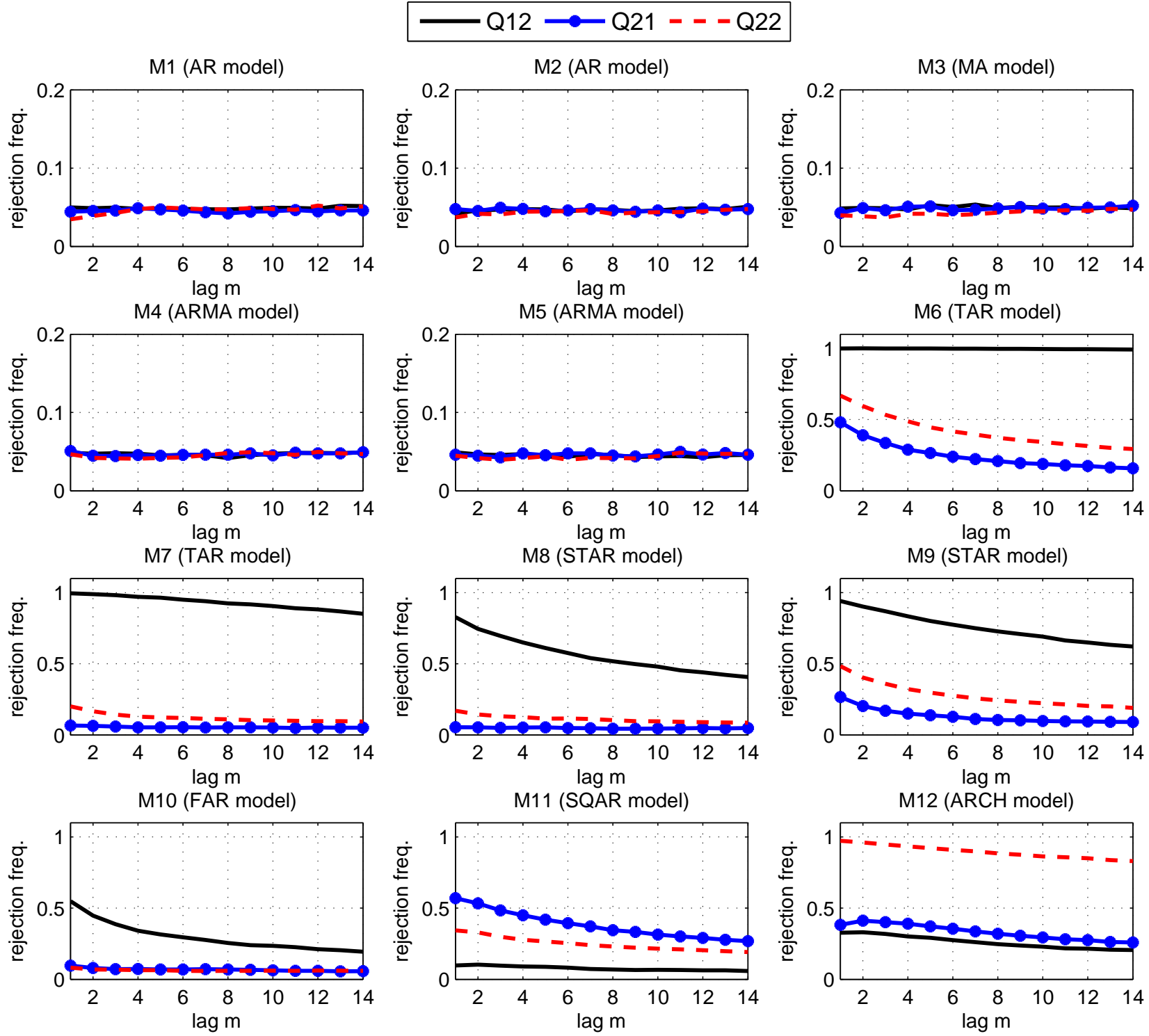

M11 (SQAR model)
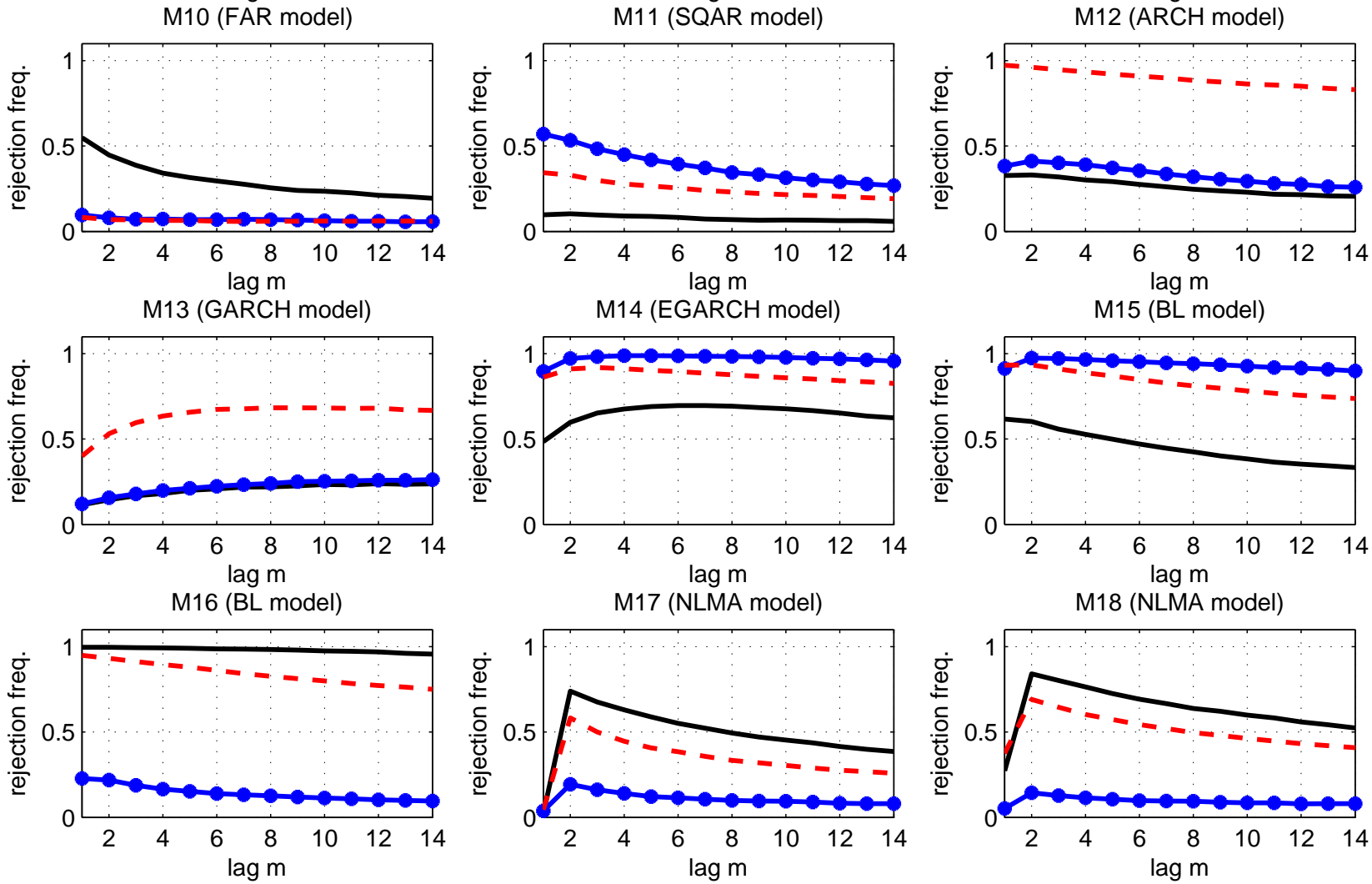

Portmanteau Tests for Linearity of Stationary Time Series 
Figure 2: Rejection frequencies of $Q_{r s}$ tests: $T=500$
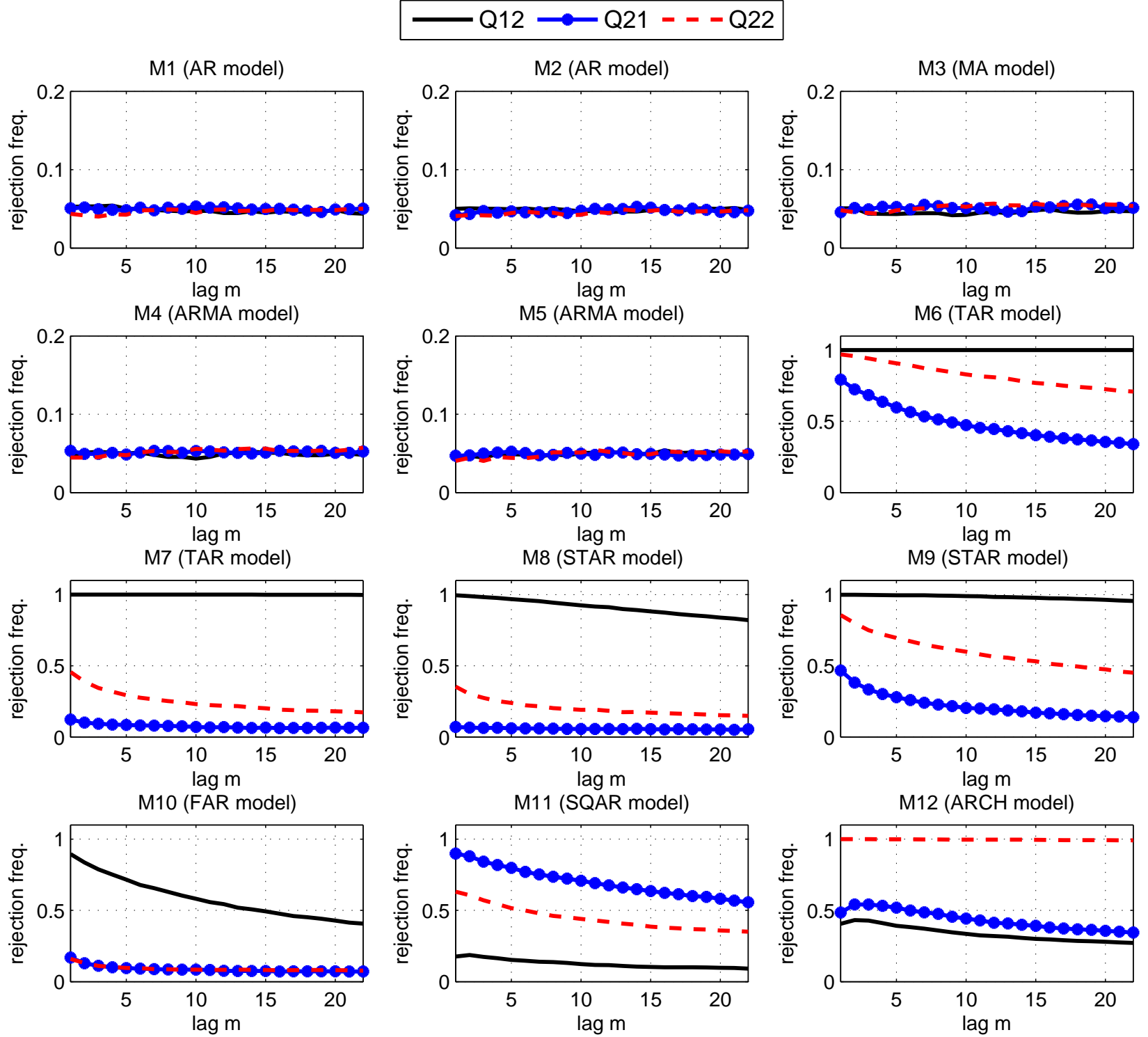

M11 (SQAR model)
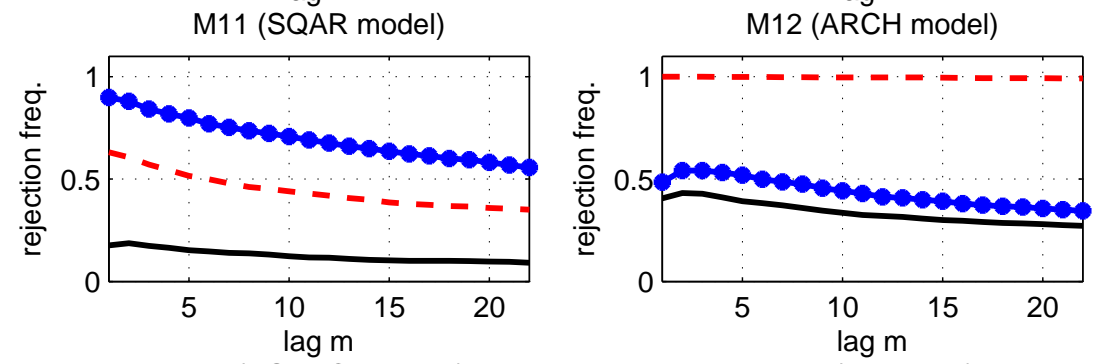

M13 (GARCH model)

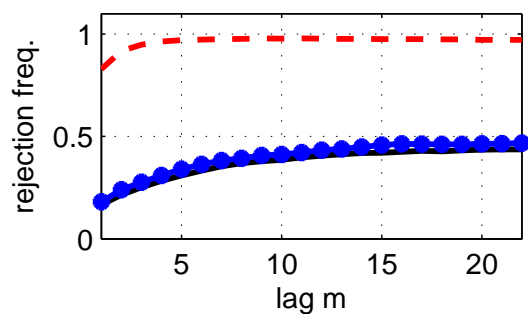

M14 (EGARCH model)

M15 (BL model)
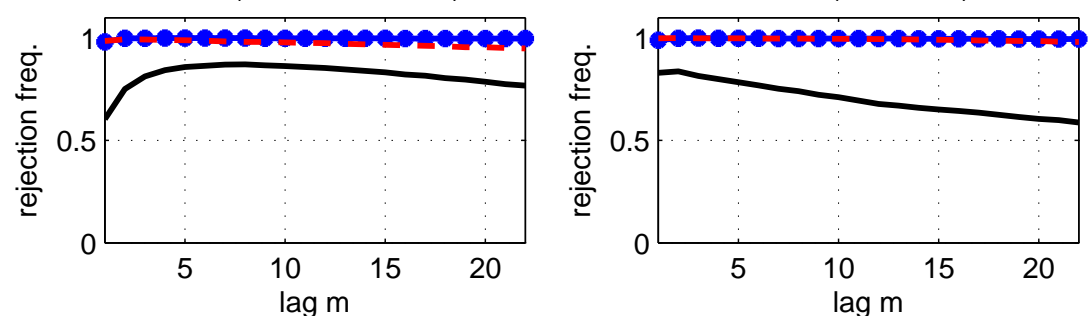

M17 (NLMA model)
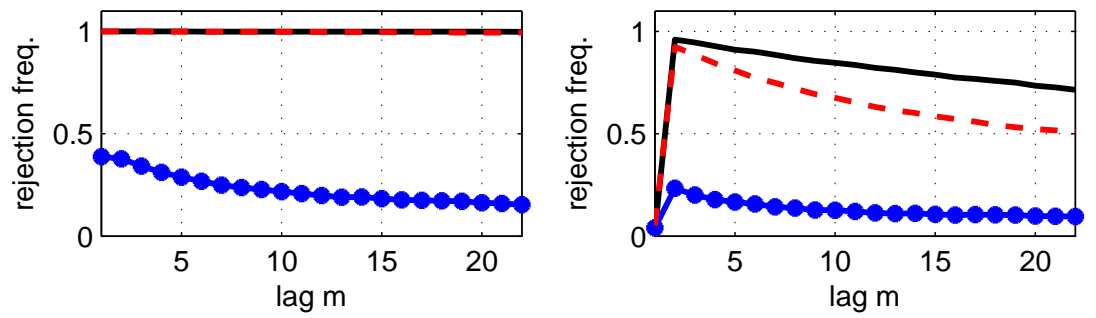

M18 (NLMA model)

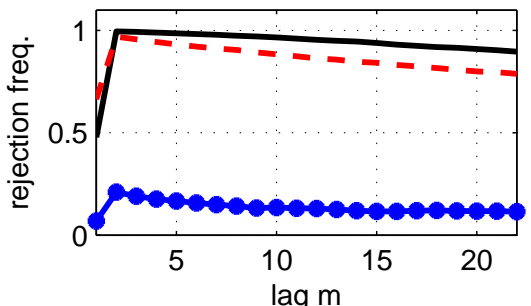

Portmanteau Tests for Linearity of Stationary Time Series 
Figure 3: Rejection frequencies of $Q_{r s}$ tests: $T=1000$
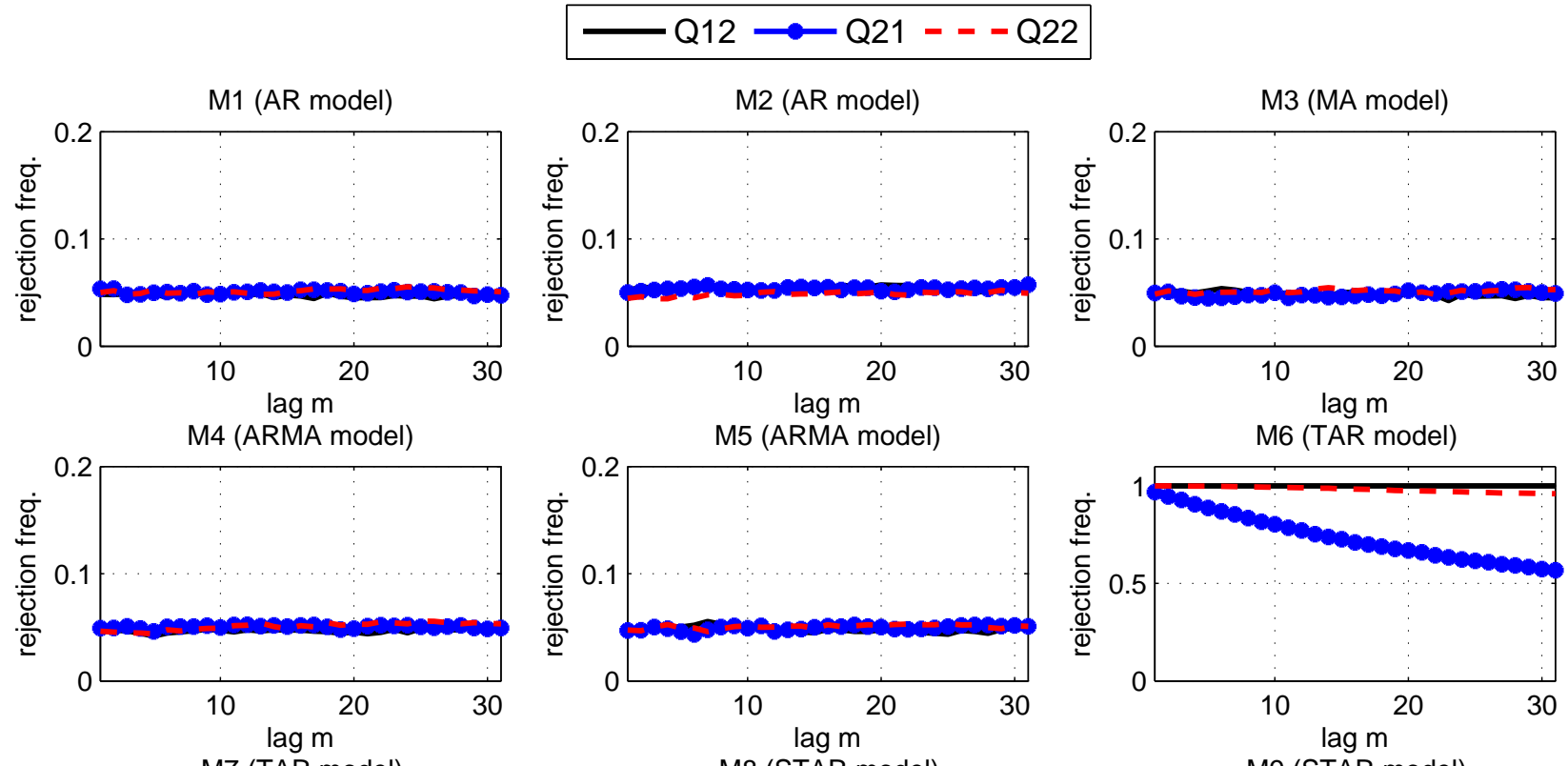

M7 (TAR model)

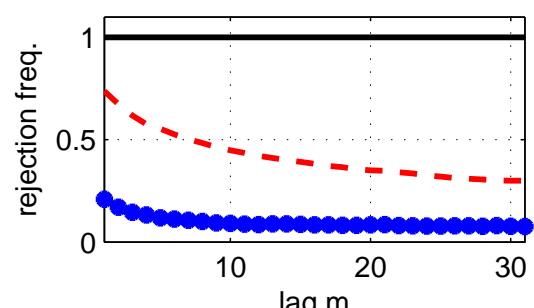

M8 (STAR model)

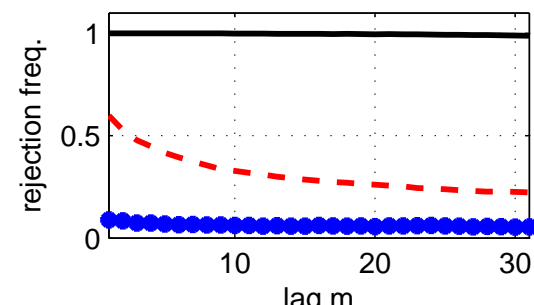

M9 (STAR model)

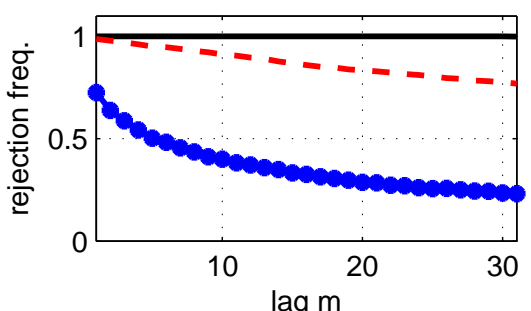

M11 (SQAR model)
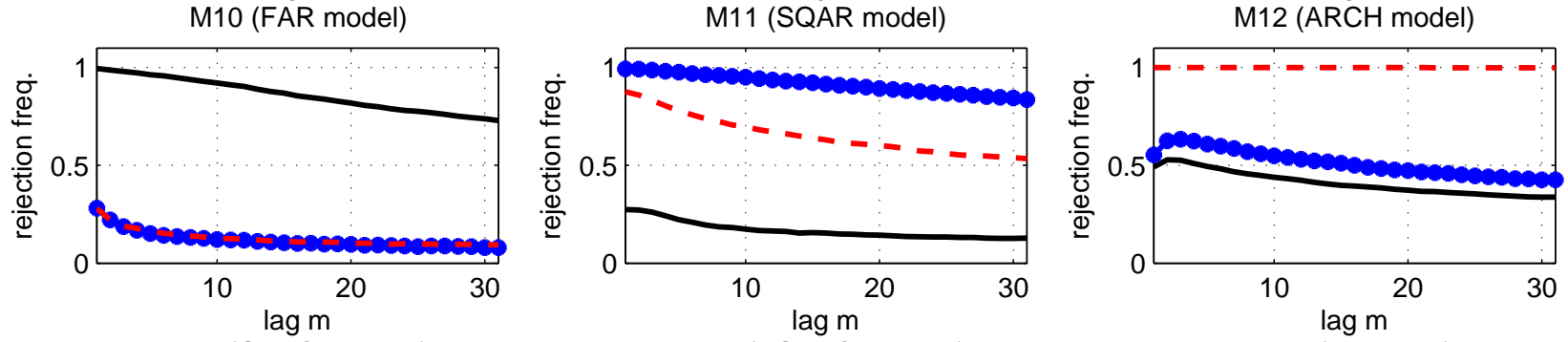

M14 (EGARCH model)
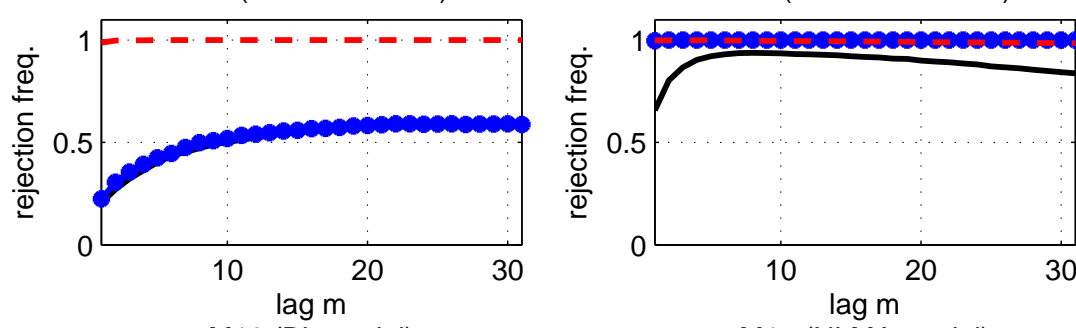

M15 (BL model)

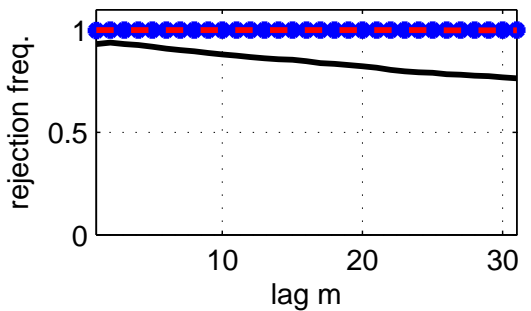

M17 (NLMA model)
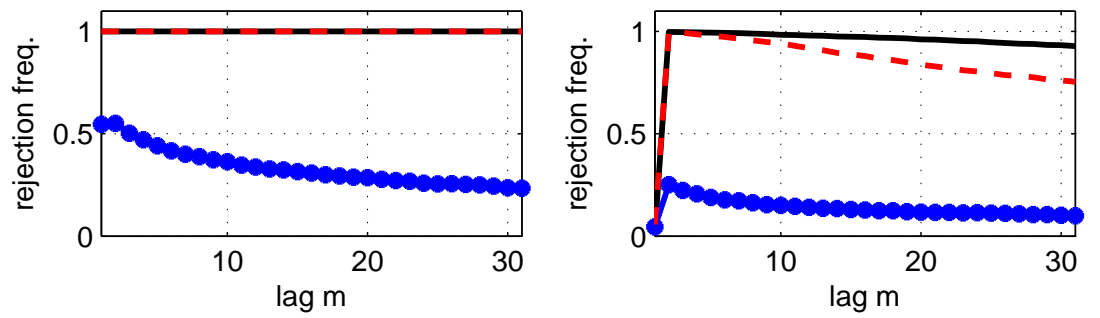

M18 (NLMA model)

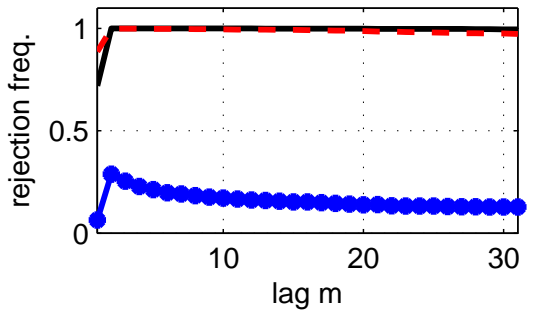

Portmanteau Tests for Linearity of Stationary Time Series 
Figure 4: Rejection frequencies of $Q_{r s}$ tests under non-Gaussian noise

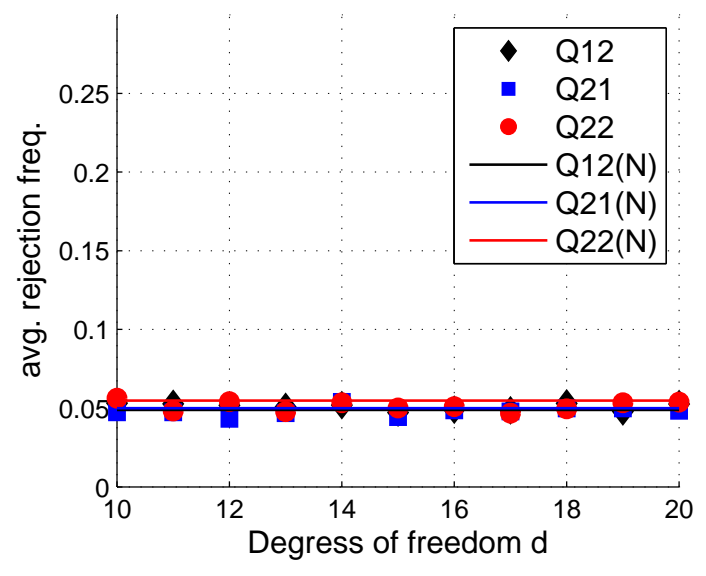

(a) Size (Student distribution)

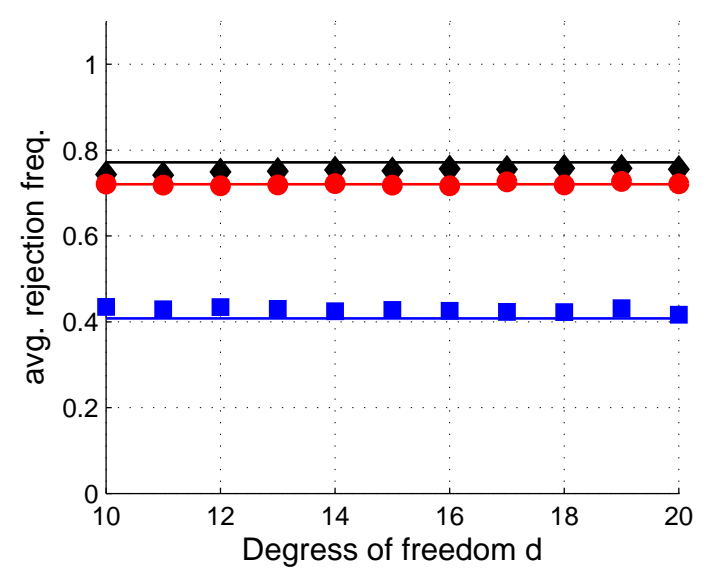

(c) Power (Student distribution)

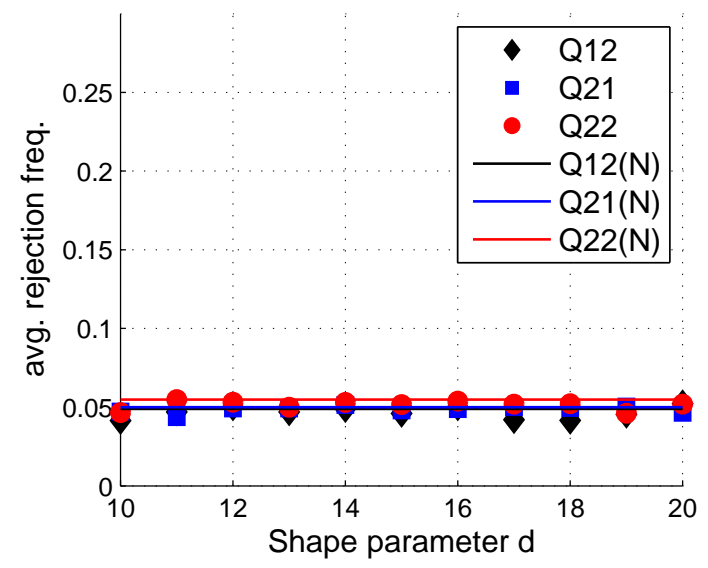

(b) Size (Gamma distribution)

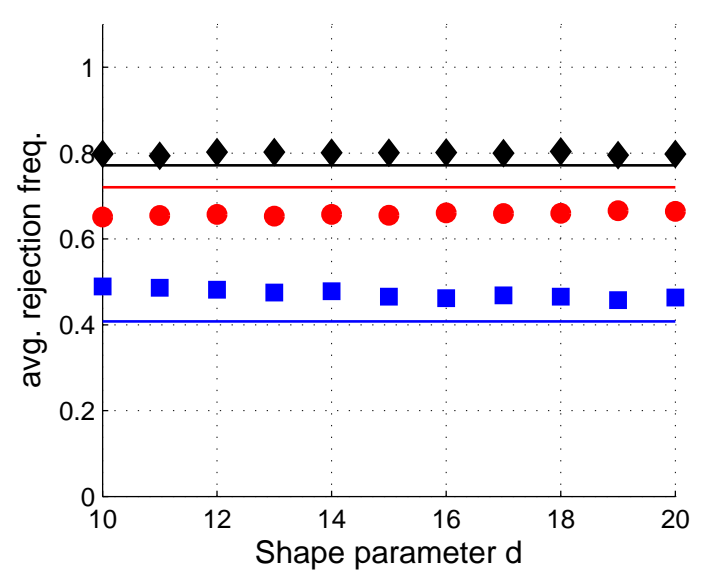

(d) Power (Gamma distribution) 
Figure 5: Rejection frequencies of $Q_{r s}$ tests under contamination

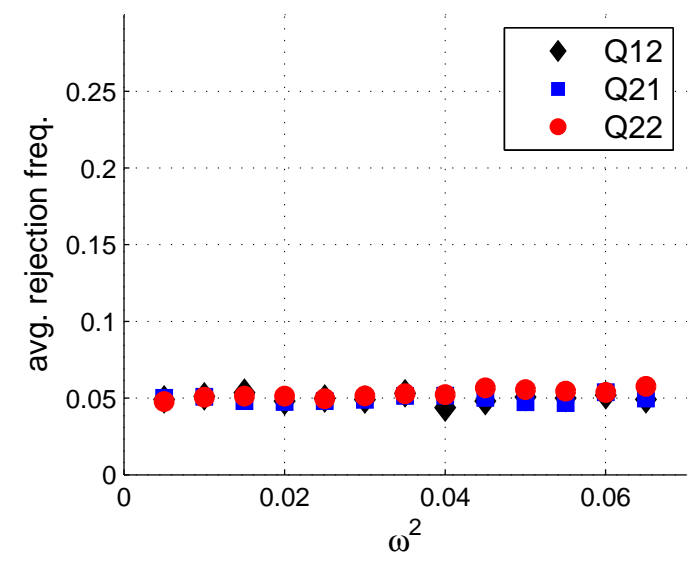

(a) Size (Student distribution)

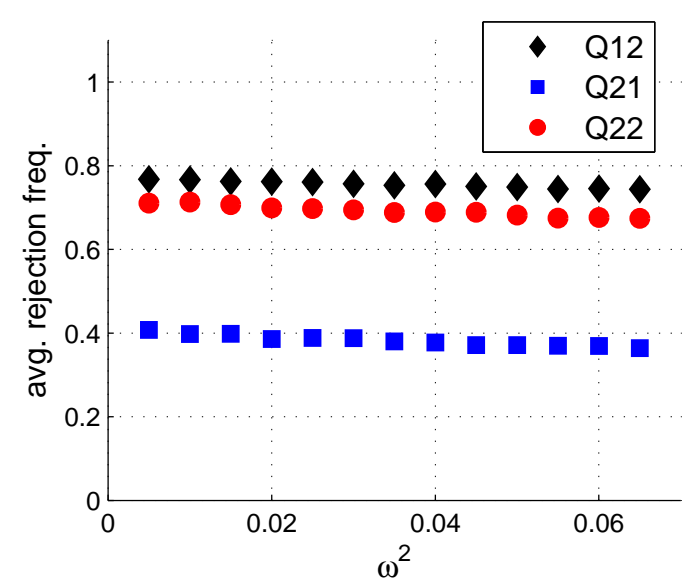

(c) Power (Student distribution)

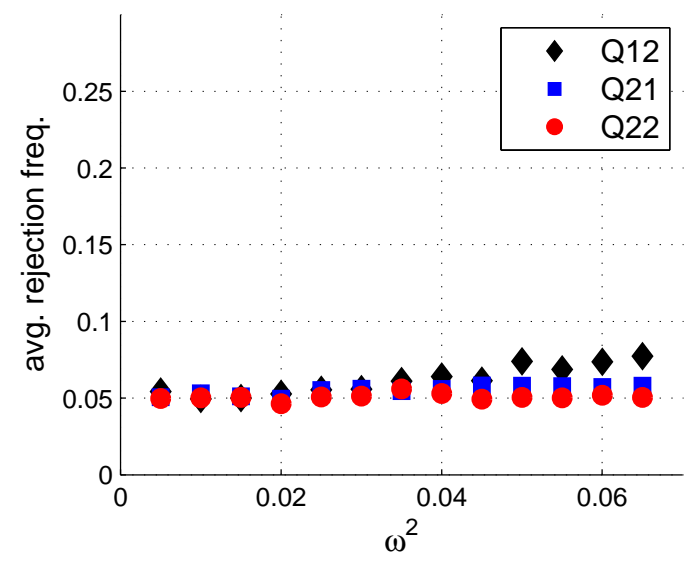

(b) Size (Gamma distribution)

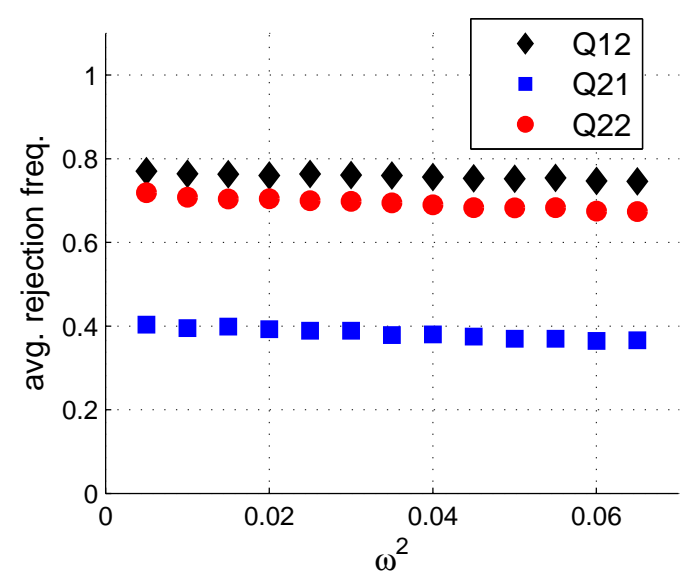

(d) Power (Gamma distribution) 